Lymphology 53 (2020) 97-98

\title{
EDITORIAL
}

\section{SARS-CoV-2/COVID-19, LYMPHATIC VESSELS, LYMPH, AND LYMPHOLOGY}

\author{
M.H. Witte, S.K. Daley
}

Departments of Surgery and Pathology, University of Arizona College of Medicine, Tucson, Arizona, USA

\section{ABSTRACT}

Lymphatic vessels and lymph are a missing link in SARS-CoV-2/COVID-19 pathophysiology and therapeutic strategies. Based on well-established principles of lymphatic function and dysfunction and a neglected literature, this article highlights promising directions for future research and clinical exploration.

Keywords: SARS-CoV-2, COVID-19, lymphatic vessels, lymph, pathophysiology, therapy

In regard to the pathophysiology and treatment of SARS-CoV-2/COVID-19 infection, blood vessels and blood/plasma have been prominently featured in leading articles in major scientific journals (1-3) and respected news media $(4,5)$. Yet not a single mention has been made of "lymphatic vessels" and "lymph" (the fluid), which are much closer to where the action is taking place in the tissue interstitium and thereby represent a missing link in understanding and therapy. Indeed, when fluid and other substances leak from damaged pulmonary blood capillaries and harmful products from reactive cells accumulate in surrounding tissue spaces, pulmonary lymphatic vessels draining air passages and oxygen-exchanging surfaces are the final common pathway (6) to remove tissue fluid "waste" containing virus and damaging generators of "cytokine storm." In an even earlier event, SARS-CoV-2 likely gains entry preferentially into lymphatic capillaries within the nasopharyngeal epithelium. Here, particles the size of free and cell-bound SARS-CoV-2 are absorbed into local lymphatics [which should be distinguishable with specific immunohistochemical stains (7)] rather than blood vessels and can continuously migrate retrograde through perineural lymphatics surrounding the olfactory bulb and cribriform plate to invade the brain substance directly. Normally, lung (and facial) lymphatics course through interposed "lymph nodes" (scarcely mentioned in relation to COVID-19 except for trafficking immune cell populations and their mobility characteristics and products) to reach two major collecting channels terminating in the neck - the left thoracic duct (TD) and right lymphatic duct (RLD), which empty into the central veins carrying lymphrich blood to the right heart, lungs (again), left heart, and rest of the body. Any potential COVID-19 vaccine with adjuvants would likely follow the same general lymphatic pathway and serial steps in the immune response following inoculation (6).

Accordingly, in severe COVID-19 cases, it should be beneficial to remove this excess volume of infected and inflammatory lymph(edema) fluid interfering with oxygen exchange (and possibly also contributing to olfactory, taste, hearing, visual, and brain dysfunction) and leading to chronic tissue 
fibrosis. Indeed, thoracic duct cannulation (TDC) with extended external drainage of lymph has been a simple, safe, and easily monitored surgical procedure performed in the neck under local anesthesia in many hundreds of patients throughout the world since the 1960's (8) continuing to the present $(9,10)$ to understand and treat a wide variety of medical conditions characterized by edema, serous effusions, inflammation, lipid dysregulation, and circulating toxins and also to immunosuppress organ transplant rejection. Specific removal of harmful lymph components (e.g., those generating "cytokine storm") and reinfusion/replacement of beneficial/ essential components (e.g., antiviral antibodies, albumin, specific immune cell populations, fluid) can be carried out selectively. Large quantities of "convalescent lymph" (several liters per day for several days) could be donated safely as a convalescent plasma (11) alternative (actually a major source) to boost immunity in seriously ill patients. Or the two procedures could be combined lymph removed and convalescent plasma replacement - in select settings. Additionally, peripheral endolymphatic antiviral drug administration (12) could reach sequestered SARS-CoV-2 lymph node "reservoirs" responsible for viral persistence and autoimmune hyperreactivity.

Furthermore, surgical procedures are no longer necessary as interventional radiologists can now access TD, RLD, and more distal lymphatics (e.g., hepatic and intestinal) by magnetic resonance imaging and other imageguided direct puncture or endovascular catheter manipulation $(13,14)$. Thus, omission of "' lymphatic vessels" and "lymph" in thinking about SARS-CoV-2 and COVID-19 not only overlooks a crucial pathophysiologic pathway but also obscures an opportunity to test several novel potentially effective strategies to ameliorate the situation.

\section{REFERENCES}

1. Mathesen, N, P Lehner: How does SAR-CoV2 cause COVID-19? Science 369 (2020), 510-511.

2. Wersinger, WJ, A Rhodes, H Cheng, S
Peacock, HC Prescott: Pathophysiology, transmission, diagnosis and treatment of Coronavirus disease 2019 (COVID-19): A review. JAMA 324 (2020), 782-793, Fig.2 p. 785

3. Ackerman, M, S Verleden, M Kuehnel, et al: Pulmonary vascular endothelialitis, thrombosis, and angiogenesis in COVID-19. NEJM 383 (2020), 120-128.

4. COVID-19: Anatomy of a Cytokine Storm" Wall Street Journal, 4/10/20, p. Al,A8 (diagram)

5. Kimberlin, K: The Treatment That Could Crush COVID. Wall Street Journal, 8/14/20 p.A 15.

6. Yoffey J, F Courtice: Lymphatics, Lymph and Lymphoid Tissue. Academic Press, 1970, pp 66-71, 158-159, 399-402.

7. Evangelou, F, P Kyzas, T Trikalinos: Comparison of the diagnostic accuracy of lymphatic endothelium markers: Bayesian approach. Mod. Pathol. 18 (2005), 490-497.

8. Dumont, A, M Witte: Clinical usefulness of thoracic duct cannulation. In: Advances in Internal Medicine. Stollerman H, ed., Vol. XV.Year Book Medical Publ., Inc., Chicago, 1969, pp 51-72.

9. Dagernier, T, N Reynaeert, G Deby-Dupont, et al: Thoracic duet drainage in the treatment of respiratory failure complicating severe acute pancreatitis. Inten. Care Med. 15 (1989), 372-378.

10. Wang, H, A Escott, K Phang, et al: Indications, techniques and clinical outcomes of thoracic duct interventions in patients: A forgotten history? J Surg Res 2018;204: 213-27.

11. Casadeval, A, M Joyner: A randomized trial of convalescent plasma for COVID-19 potentially hopeful signal. JAMA 324 (2020), 455-457.

12. Trevasbus, N, L Kam, M Rai, C Porter: From sewer to savior: Targeting the lymphatic system to promote drug exposure and activity. Nature Reviews 14 (2015), 782-803.

13. Itkin, M, G. Nadolski: Modern techniques of lymphangiography and intervention. Cardiovasc. Intervent. Radiol. 41 (2018), 366-376.

14. Schwartz, F, O James, P Kuo, et al: Lymphatic imaging: Current noninvasive and invasive techniques. Semin. Intervent. Radiol. 37 (2020), 1-13.

Marlys H. Witte, MD

Professor of Surgery, Neurosurgery, \& Pediatrics University of Arizona College of Medicine 1501 N. Campbell Ave., PO Box 245200

Tucson, Arizona 85724-5200 USA

E-mail: lymph@surgery.arizona.edu 